\title{
Antibody based affinity capture LC-MS/MS in quantitative determination of proteins in biological matrices
}

Trine Grønhaug Halvorsen, Léon Reubsaet*

Department of Pharmaceutical Chemistry, University of Oslo, Oslo, Norway

*Corresponding Author: Léon Reubsaet, P.O. Box 1068 Blindern, NO-0316 Oslo,

Norway, e-mail: leonr@farmasi.uio.no Phone: +4722856613, Fax: +47-22854402 


\section{Abstract}

Determination of proteins in complex biological matrices has massive attention and is exploited in many different scientific disciplines. Routinely, proteins are determined using antibody based immuno-metric assays. Although these assays are easy to perform and widely used, interpretation of the results is challenging: cross reactivity, high dose hook-effect, presence of heterophile- or auto-antibodies give rise to false results, sometimes with dramatic consequences. In the quest for more robust assays a combination of antibody sample clean-up, tryptic digestion and mass spectrometric determination is gaining more attention. This review discusses the advantages of antibody based affinity capture and subsequent LCMS/MS in protein analysis like less false results and possibilities like multiplexing and isoform differentiation. It also considers the interplay between the analytical, biological and biochemical factors, which still give rise to false results, even with mass spectrometry as the ultimate selective detection step. The intention of this review is to point out both strengths and weaknesses of antibody based affinity capture LC-MS/MS in quantitative determination of proteins in biological matrices.

Keywords: immuno-capture; antibody; bottom-up proteomic strategy utilizing LC-MS/MS; protein quantification; false results. 


\section{Introduction}

Historically the interest in, and importance of reliable protein quantification is shown by the attention method development by Lowry et al. and Bradford has gained. Both methods describe colourimetric measurements of the total protein content in the $\mu \mathrm{g} / \mathrm{mL}$ scale and are within the top 3 of most cited research papers all times (more than 450000 citations together [1] in 2014). The last paper in this top 3 was written by Laemmlli and descirbes protein separation using SDS-PAGE. Since the early 1950's the field of protein analysis evolved from total protein concentration determination and SDS-PAGE based separations (in the $\mu \mathrm{g} / \mathrm{mL}$ scale) to complex multidimensional proteomic analyses allowing to separate thousands of proteins and to determine concentrations of certain proteins down to fg levels (and lower). These biochemical and analytical efforts revealed that there is an enormous variation in the type of proteins, amount of isoforms, post translational modifications, function and much more. Beside their biological function, many proteins have, by means of their concentration level in blood diagnostic value as well as they might, by means of their presence in certain samples, give insight in biochemical processes in the body.

Although there is an overwhelming variation in the biochemical and analytical approaches to proteins, targeted protein quantification using antibody based affinity materials together with a bottom-up proteomic strategy utilizing LCMS/MS analysis is gaining much interest in clinical chemistry (proteins with diagnostic value), therapeutic drug monitoring, drug discovery and development of new drugs (protein biopharmaceuticals), medicine (explorative, revealing biochemical processes) and sports (doping analysis). This overview will mainly deal with the interplay between the analytical and biological aspects of antibody 
based affinity materials combined with a bottom_up proteomic strategy utilizing LC-MS/MS analysis. It discusses the possibilities immunocapture LC-MS/MS gives for quantification and understanding biochemical processes as well as their limitations. It will deal with targeted applications for clinical diagnostics and protein drug analysis where triple quadrupole and iontrap due their robustness, cost and availability are the mass spectrometers of choice. It will not deal with the relative quantification (e.g. SILAC, iTRAQ, ${ }^{18} 0$ labelling) of the proteome, nor will it deal with DDA analyses to reveal protein modifications. Generic capture strategies for the enrichment of post translational modifications like ubiquitination, glycosylation and phosphorylation are not discussed.

\section{Why combining antibody based affinity with bottom-up proteomic strategy utilizing LC-MS/MS?}

Proteins are diverse in their biological function, and only 20 different amino acids make up for most of the protein variation. Additionally, proteins exhibit a chemical variability caused by, among others, their length, hydrophobicity, side chain $\mathrm{pK}_{\mathrm{a}}$ values, $\mathrm{pI}$ values and post translational modifications. Proteins are not easy to separate from each other using conventional pre-analytical sample treatment. Mass spectrometry offers outstanding sensitivity, selectivity and ability to determine many substances simultaneously. These are some of the reasons why MS presently is frequently used in protein analysis. However, the broad range of which proteins occur in biological samples may give rise to analytical challenges.

Human plasma is mainly used as biological matrix for protein determination and contains many proteins in different concentrations: albumin and transferrin have 
reference levels in the medium $\mathrm{mg} / \mathrm{mL}$, proteins like C-reactive protein in the low $\mu \mathrm{g} / \mathrm{mL}$ range while interleukin-6 occurs in the low $\mathrm{pg} / \mathrm{mL}$ range [2]. In a bottomup approach (i.e. enzymatic cleavage of the protein prior to analysis) it is not possible for mass spectrometers to determine proteolytic peptides from for instance the low abundance interleukin-6 (low pg/mL level) with high precision and accuracy in the presence of proteolytic peptides from the high abundance protein albumin (medium $\mathrm{mg} / \mathrm{mL}$ level). This is due to the limited dynamic range of the mass spectrometer [3], which can defined as the ratio between the highest and lowest reliable concentration measurement in the same analysis. In 2006, Anderson and Hunter [4] showed that the dynamic range of an MRM assay for more than 50 high and medium abundant plasma proteins with minimal pretreatment of the plasma sample was 4.5 orders of magnitude allowing detection to sub $\mu \mathrm{g} / \mathrm{mL}$ level. To allow determination of lower levels of a target protein in complex samples other approaches need to be applied. Among these multidimensional chromatographic separations (like MudPIT [5]) and depletion of the most abundant proteins (depending on the vendor one can deplete for up to more than ten abundant proteins) might be used. These techniques have a tremendous value in the field of exploratory proteomics they have the major setback of being time ineffective when proteins need to be determined for diagnostic purposes. In the latter case a high analytical throughput is of importance. Regardless the above-mentioned analytical approaches, the limit of detection (and quantification) for proteins in complex samples using LC-MS/MS remains at its best in the $\mathrm{ng} / \mathrm{mL}$ level, and is thus with respect to sensitivity not competitive with immunological assays. A logical question would be why immunological assays need to be replaced by MS based methods. 


\section{Drawbacks in immunological methods for protein analysis}

In routine determination of endogenous proteins in hospital laboratories, the methods of choice are often based on immunological principles, ranging from ELISA to RIA. After their introduction in the 1950-1960s, they gained popularity due to their relative simple, sensitive and robust analytical protocols. Although these methods are based on the use of specific antibodies, they have shown to have many drawbacks potentially giving rise to false diagnosis caused by factors like presence of auto-analyte antibodies, heterophile antibodies or human antireagent antibodies (like human anti-mouse), cross reacting reagents and high dose hook effect[6]. Another drawback is the lack of differentiating ability: multiplexing several diagnostic markers to enable more robust diagnosis mostly needs several analyses (one analysis per diagnostic marker), additionally, no subtyping or differentiation between isoforms or isovariants of a certain biomarker is not possible within one single analysis.

\section{Combining immunoaffinity with mass spectrometry}

As already mentioned, to be able to determine proteins of interest in complex biological samples by mass spectrometry at fg - pg levels clean-up and/or enrichment is needed. There are several generic ways to perform this, but the possibilities antibodies offer in term of sample clean-up are outstanding. A combination of the advantages of the mass spectrometer as well as the advantages of the use of antibodies in targeted protein determination is reported as early as 1996 by Liu and Bowers[7]. In this work human chorionic gonadotropin (hCG) is trapped by immunoaffinity from urine followed by tryptic digestion and mass 
spectrometric detection of its proteotypic peptide. It took almost 10 years for a similar combination strategy was published (also on urinary hCG)[8] but after that, more target proteins were determined by combining immunoaffinity, proteolysis and LC-MS/MS for diagnostic purposes[9], doping related analyses[10] and bioanalysis of biopharmaceuticals[11]. The combined strategy not only allows to determine very low protein concentrations, it additionally allows to reveal and monitor protein isoforms and variations[12].

In general the workflows described here and in the following examples are divided in the protein capture approach and the peptide capture approach[13]. (see Figure 1)

In the following chapters a critical overview is given of the work done in the field of immunocapture LC-MS/MS for targeted determination of bioactive proteins. Although work has been done on the top-down analysis of proteins like insulin, IGF1 and other hormones, this review is limited to protein determination after bottom-up analysis.

\section{Antibodies: the essential ingredient}

As mentioned earlier, the immuno-capture step can either be selective capture of intact proteins (placed prior to the digestion step) or selective capture of proteotypic peptides (placed after the digestion step. SISCAPA: see later). Either way, the antibody is essential for a well functioning method. In the circumstances were several antibodies against a target protein are available, selection can simply be carried out by evaluating their performance $[14,15]$ with respect to amount of antigen found in the bound fraction vs. antigen found in the unbound fraction. 
Such evaluation using LC-MS/MS is much less time-consuming than an evaluation using conventional immunoassays.

\section{Anti-protein antibodies}

Many reports are on LC-MS methods with the immuno-capture step before the digestion where antibodies against intact proteins are used. Using this concept, the MS detection will avoid possible false positive results caused by crossreactivity. However, the use of antibodies against intact proteins in immunocapture LC-MS/MS still will have some of the potential drawbacks seen in immunoassays hampering reliable quantification of target proteins: Very high concentrations of antigen may not lead to a hook effect, but it might challenge the capture capacity thus lead to false low results. (this can be corrected for using a labelled protein standard which exhibits the same affinity as the target protein). Additionally, auto-analyte antibodies or anti-reagent antibodies present in the biological samples will to the same extent as in immunoassays lead to false low results.

\section{Anti-peptide antibodies}

Anti-peptide antibodies, known as the SISCAPA approach (Stable Isotope Standards and Capture by Anti-Peptide Antibodies) was firstly described by Anderson et al.[16] and thoroughly reviewed by Becker and Hoofnagle [17]. In this approach, polyclonal anti-peptide antibodies are raised against the already chosen proteotypic peptides of target proteins. Compared to antibodies raised against whole proteins the affinity of anti-peptide antibodies has been described to be somewhat lower[18]. The analytical advantage is that stable isotope peptides can correct for variations in the capture step as stable isotope peptides are captured to the same extent as proteotypic peptides from the endogeneous 
proteins. Another advantage is the ability to reuse SISCAPAs antibodies as they are introduced after proteolysis[19]. Additionally compared to the use of antiprotein antibodies, presence of auto-analyte or anti-reagent antibodies will to a much lesser extent influence on the quantification of a target protein. A disadvantage is that isoform differentiation of the target protein is less, if at all, possible with the use of a SISCAPA.

\section{Affinity formats}

There are many formats and platforms used for the capture of the target protein or peptide. A comprehensive review discussing advantages and shortcoming of the different formats/platforms is published by Li et al.[20] in this journal and will thus not be covered here. No matter the choice of the format or support, materials used for the immobilisation should be inert. However, there will always be proteins and other compounds binding to them. This non-specific binding should preferably be eliminated as it might cause ion suppression of very low abundant target proteins thus hampering satisfactory low quantification limits. Removal can be carried out either before the affinity extraction or post-analysis by substracting the signal of the contaminant from the obtained spectra[21].

In choosing the right format of the support to attach the antibodies, one should bear in mind quantification limits needed as well as the labour intensiveness of the method. The 96-well plate format for extractions is well suited for integration in many analytical platforms, thus automatable and diminishing the labour intensity[22]. One of the disadvantages is the limited capacity of the wells: the active surface is low compared to beads. Another disadvantage, compared to the use of beads is the limited possibility for enrichment: The maximum amount of 
sample $(200 \mu \mathrm{L})$ might be reduced to $50 \mu \mathrm{L}$ in wells (enabling up to 4 times enrichment). Using beads, the sample amount is not limiting, in other words, $1 \mathrm{~mL}$ of sample can easily be extracted and then enriched to $50 \mu \mathrm{L}$ (enabling up to 20 times enrichment)[23].

\section{Advantages and possibilities of immuno-capture LC-MS}

Combining the immuno-capture step with mass spectrometric detection does not resolve all the limitations seen in the methods separately, however, it has his advantages as well as it opens for new possibilities.

\section{Epitope mapping}

The first description of immunocapture MS was the use in epitope mapping based on affinity capture of proteolytic peptides. As a first step proteolytic peptides were generated, these peptides were in the next step captured by immobilized antibodies (raised against the whole protein) followed by a washing step to remove unbound proteolytic peptides. After elution, the mass spectrometer was used to identify the bound peptides thus identifying the epitope. In this way epitope containing peptides for proteins like melittin and glucagon-like peptide[24] were identified. A slight different approach was chosen by Macht et al. who allowed coupling of the whole protein (cardiac troponin) to the antibody prior to proteolysis. Using limited proteolysis (trypsin:protein ratio below 1:100) the epitope was excised: the epitope containing part remains attached to the antibody during the proteolysis while the other parts of the protein were released. After elution, the epitope containing peptide was determined using mass spectrometry[25].

Multiplexing using one antibody 
In a review of Kiernan, the main strength of immuno-capture followed by LC-MS was described to be that isoforms and protein modifications might be determined in a single step [26]. Since the (reagent) antibody will interact with only a relative small part of a protein, changes in the target protein, which are neither within nor close to the epitope region, will not affect the immune-reactivity of the reagent antibody. This allows capture of target protein isoforms and classes of proteins (figure 2a). An example, which supports this, is the immuno-capture LC-MS analysis of pro-gastric releasing peptide (ProGRP). This protein has been studied thoroughly in various studies since ProGRP is an acknowledged marker for Small Cell Lung Cancer. It is routinely determined by an immuno-metric assay and was demonstrated to consist of three isoforms when determined by immuno-capture LC-MS. Although only the total amount of ProGRP is used for diagnosis, it is clear that there is a diagnostic potential in differentiation of the ProGRP isoforms using immuno-capture LC-MS[27]. Other examples of simultaneous immuno-capture and analysis of related proteins/peptides exhibiting the same epitope are determination of $20 \beta$-amyloid peptide signatures[28], three tau proteins in human cerebrospinal fluid (CSF) [29], and up to 8 variants of hCG in human urine and serum [30]. The quantification of 1-84 parathyroid hormone in patient samples at pM levels by immuno-capture LC-MS showed its superiority over conventional immunoassays with respect to the ability to distinguish and determine C-terminal metabolic peptide products[31]. In addition to these examples of endogenous proteins, seven different antibody therapeutics with immune reactivity for a single (reagent) antibody are described[12].

Multiplexing using several antibodies in the extraction step 
Combinations of several (reagent) antibodies in a single analysis are also described. In this case several batches of immobilized (reagent) antibodies are mixed before the immuno-capture step. This mixture is then used to capture several target proteins in a single step (figure $2 \mathrm{~b}$ ). For multiple extractions at the protein level this is described up to capture of six proteins: In a study of Nicol et al. five (reagent) antibodies against carcinoembryonic antigen (CEA), secretory leukocyte peptidase inhibitor (SLPI), tissue factor pathway inhibitor (TFPI), tissue factor pathway inhibitor 2 (TFPI2) and metalloproteinase inhibitor 1 (TIMP1) were immobilized separately to beads and combined to allow a one-step extraction and determination of these lung cancer markers [32]. Lin et al. [33] combined six different antibodies to capture metalloproteinase inhibitor 1 (TIMP1), cartilage oligomeric matrix protein (COMP), thrombospondin-2 (THBS2), endoglin (ENG), mesothelin (MSLN) and matrix metalloproteinase-9 (MMP9) in a single extraction and analysis. These data was compared to data from ELISA experiments for each of the proteins and showed close correlation. A combination of both multiplexing and isoform differentiation was described for the isoforms of the lung cancer marker ProGRP and NSE where only two (reagent) antibodies were used [34]. This single analysis improves diagnostic robustness. In all the above cases (reagent) antibodies against the target protein were used. This multiplex strategy can alo be applied by using the SISCAPA approach $[35,36]$. Ippoliti et al. even showed automated multiplex extractions of more than 150 peptides[37].

The use of antibodies generated from libraries

The above mentioned approach is based on antibodies with proven specificity and applicability (for instance from immunoassays like ELISA). In the quest for 
(reagent) antibodies multiplex immuno-capture LC-MS experiments can be carried using batches of antibodies generated from antibody libraries are used for capture purposes: motif-specific antibodies from the nCoDer library coupled to magnetic beads are mixed to capture classes of proteins. In such a manner Olsson et al. reduced the complexity of the yeast proteome down to 75 motif contain proteins, some of them expressed as low as only 50 copies per cell[38]. Whiteaker et al. used antibody fragments (Fabs) generated from the HuCAL PLATINUM library to capture only epitope containing tryptic peptides of the target proteins[39].

The advantages with the multiplexing approach mentioned in this section lead to the ability to determine several biomarkers simultaneously shorten the response time (instead of five separate analyses, only one needs to be performed), there is less room for analytical and operator errors, making the diagnosis more robust. Additionally, one can ad-hoc mix immobilized reagent antibodies to produce a new immuno-capture procedure allowing for personalized biomarker analysis

It must however be stressed that this only is the case when all these different variants of the target protein have sufficient immuno-reactivity.

\section{Limitations and sources of variability in target protein quantification by immuno-capture LC-MS}

Besides general pre-analytical variations like sample handling, sample collection and temperature as well as general analytical variations like injection reproducibility, mass spectrometric response and matrix effects reviewed by 
Percy et al.[40] there are also biological and biochemical factors influencing the analysis of target proteins by the bottom-up approach.

\section{Variability of digestion: impact on quantification}

The choice and origin of the proteolytic enzyme affects the outcome of the quantitation of the target protein[40]. There are numerous of proteolytic enzymes which can be chosen for this purpose, all with their shortcomings (like challenging cleavage sites) and advantages (like degree of efficiency). This is reviewed by Giansanti et al.[41]

Variability in proteolysis of the target protein might be corrected for by using a suited internal standard. One should be aware that isotopically labelled proteotypic peptides will not correct for these variations, neither when antiprotein antibodies are used nor when anti-peptide antibodies are used. The 'ideal' internal standard which corrects for proteolytic variations (as well as the capture by the antibody) is the PSAQ approach (Protein Standard Absolute Quantification) which are isotopically labelled versions of the target proteins[42]. Another intermediate approach is shown by Faria et al.[43] who studied the quantification of human osteopontin by application of an extended stable isotope labelled internal standard (TYDR $\underline{\left.G S D V V^{*} Y G L R S K S K K F\right)}$ containing cleavage sites on both $\mathrm{N}$ - and C-terminal of the chosen proteotypic peptide (GSDVVYGLR). The internal standard was added after the immuno-capture step (use of anti-protein antibody) but before the tryptic digestion. It showed that the extended internal standard improved the robustness of the quantification by both correcting for trypsin variability as well as peptide instability. Both PSAQ and the extended isotopically labelled internal standard would also work for immunocapture strategies using anti-peptide antibodies. 
Modifications of the target protein affecting the immuno-reactivity (post translational/mutations)

Selection of the reagent antibody for capture during method development is essential. This was shown by Guy et al.[44] who studied the impact of antibodies directed against various epitopes of a target protein using mass spectrometric detection (this is of course also a challenge in immunometric assays). In the determination of cardiac troponin 1, post-translational phosphorylation to its mono- and di-phosphorylated form gave rise to a change in immunoreactivity of the target protein resulting in decreased signal intensities for all tested antibodies (see figure $3 \mathrm{a} / \mathrm{b}$ ). Changing one amino acid in the epitope even caused absence of signal for cardiac troponin 1 for one of the studied antibodies thus pointing out the importance of antibody selection in the method development. [44]

Modification of the target protein affecting the production of proteotypic peptides Modifications (like post-translational modifications, mutations and degradation) in the proteotypic peptide leads to a change in $\mathrm{m} / \mathrm{z}$ and often also in the retention time which makes it potential challenging to determine the target protein by means of LC-MS/MS.

One example is endogenous protease induced changes in the target protein that will affect the production of proteotypic peptides: Streng et al. [45, 46] showed the effect of in-sample proteolysis and methionine oxidation on the determination of cardiac troponin 1 by immunocapture followed by gel separation of the captured cardiac troponin 1 degradation products and the analysis of 18 peptides after ingel digestion. As much as five different bands for cardiac troponin 1 were identified, but only one proteotypic peptide (located in a stable region) was able to reflect the original amount of cardiac troponin 1 in the sample. 
Occurrence of proteotypic peptide modifications demonstrates that the choice of the proper proteotypic peptide for the quantitative analysis in some cases is crucial and exceptional demanding (see figure 3a/c). Software like Skyline (https://skyline.ms/project/home/begin.view?) can be a very helpful tool in this respect.

Considering the above it is recommended that, if possible, at least two proteotypic peptides should be used for the quantification of the target protein. This will in general lead to a more robust analysis method and reveal challenges or pitfalls in the method.

\section{Auto-antibodies and anti-reagent / anti-drug antibodies}

Another challenge in the affinity based extraction and following determination of proteins in biological matrices is the presence of auto-antibodies, anti-reagent antibodies or anti-drug antibodies (ADA) (see figure 3a/d). As with immunoassays presence of these antibodies might lead to false low or even false negative results as the segment of the protein important for the affinity extraction (epitope) might be shielded by these antibodies. To our knowledge there is only one report describing the impact of auto anti-reagent or auto anti-drug antibodies on the bottom-up proteomic strategy utilizing LC-MS/MS determination of a target. However, the efforts to circumvent this by using DMSO to prevent ADA's effect on the determination of therapeutic mAbs. These efforts where without success[47].

Auto-antibodies also might shield the epitope which is used by the assay

antibodies as in immuno-metric assays (see figure 3a/e). Thyroglobulin concentrations in plasma are used to monitor the success of thyroid cancer treatment but the assay outcome might be hampered by the possible presence of 
auto-antibodies. In such a case, immuno-capture LC-MS using anti-thyroglobulin (reagent) antibodies will not be a better alternative. However, by using the antipeptide antibody approach and an antibody towards the specific tryptic peptide (VIFDANAPVAVR) of thyroglobulin the challenge of auto-antibodies is circumvented as the capture step is placed, in time, after the digestion step [17, $48,49]$.

\section{Subunits}

Target proteins consisting of subunits can be particularly challenging: in addition to variations occurring on the primary protein structure level (mutations, post translational modifications) also variations on the quaternary protein structure can occur through degree of association/dissociation of subunits. The much studied case of human chorionic gonadotropin (hCG) immuno-capture LC-MS gives insight in the challenges caused by this: hCG occurs as a hetero-dimer $(\alpha-\beta$ subunits) as well as a monomer (free $\beta$-subunit) and the clinical relevant variations in the hCG occur in the $\beta$-subunit (both in the hetero-dimer as in the monomer). Although the variations on the primary protein structure level can nicely be detected by determining their proteotypic peptides in the bottom-up approach, it will not be possible to determine if a particular proteotypic peptide originates from the $\beta$-subunit in the hetero-dimer or from the $\beta$-subunit in the monomer (variations in the quaternary protein structure). Sequential extractions for hCG were developed to enable determination of variations in the primary protein structure and quaternary protein structure in urine [50] and serum samples [30]. In the urine example first the monomers were extracted followed by the intact heterodimer, in the serum example the order of extraction was 
reversed. In these studies, both extracts were then treated and analysed separately.

\section{Conclusion}

Immuno-metric assays are widely used but have several disadvantages. Many of these disadvantages are dealt with using antibody based affinity bottom-up proteomic LC-MS/MS.

The reviewed approach is either performed using peptide extractions (SISCAPA) or protein extractions. In both cases a proteolytic step is needed, both allow multiplexing and both can potentially rule-out false results caused by cross-reactivity. Protein extractions and peptide extractions have complementary advantages; a clear advantage is that anti-protein reagent antibodies capture isoforms of the target protein (if the epitope is situated in a constant region) allowing isoform differentiation through mass spectrometry. On the other hand, the presence of auto-antibodies which causes false negative results (in both immuno-assays and immuno-capture using anti-protein antibodies) is potentially circumvented using peptide extractions. Both extraction methods allow more than sufficient clean-up and enrichment compared to generic sample preparation techniques enabling protein quantification at ultra low protein concentrations.

Modifications of the protein in the epitope region or in the proteotypic peptide region might lead to false negative results either through limited capture or loss of signal through change in the mass of the proteotypic peptide, using both the protein and the peptide extraction approach. Although it is dependent on the protein of interest if antibody based affinity bottom-up LC-MS/MS is the best 
alternative (compared to immunometric assays), for many proteins it has shown to improve both the quantitative and qualitative results. 


\section{Legend to the figures:}

Figure 1:

a) Sample preparation order: protein capture - proteolysis - LC-MS: the target protein is captured by an antibody which is immobilized on a support. After removal of the unbound fraction by washing, the target protein is digested into proteolytic peptides. The amount of the target protein is then determined by means of one or several proteotypic peptides using LC-MS.

b) Sample preparation order: proteolysis - peptide capture - LC-MS: the sample is digested in its whole into proteolytic peptides. The proteotypic peptides of the target protein are captured by antibodies (SISCAPA) immobilized on a support. After removal of the unbound fraction, the proteotypic peptide is released and determined using LC-MS.

Figure 2:

a) Capture of isoforms or isovariants by anti-protein antibodies, which contain a common epitope. After proteolysis proteotypic peptides for each of the captured proteins are determined simultaneously by LC-MS/MS.

b) Multiplex capture of various target proteins using a cocktail of antibodies (either anti-protein or anti-peptide antibodies). The extraction occurs in one single step. After proteolysis proteotypic peptides for each of the target proteins are determined in a single LC-MS/MS analysis.

Figure 3:

Effect of target protein modification and presence of auto-antibodies on antibodybased protein determination using mass spectrometry. 
a) Common situation: target protein is captured by antibody and proteotypic peptide is determined by LC-MS/MS after proteolysis b) Modification in the epitope affecting the immunoreactivity of the target protein c) Modification in the proteotypic peptide amino acid sequence. Target protein exhibits the same immunoreactivity but does not yield the $m / z$ of the proteotypic peptide after proteolysis d) Auto-antibodies/ anti-drug antibodies attached to the epitope or to the vicinity of the epitope will cause loss of or less immunoreactivity of the target protein e) Anti-reagent antibodies might interfere with the reagent antibody hampering the capture of target protein 


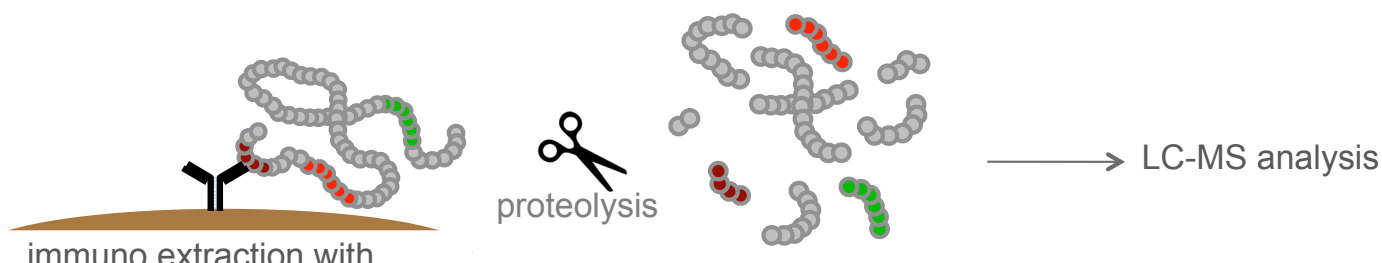

immuno extraction with

anti-protein antibody
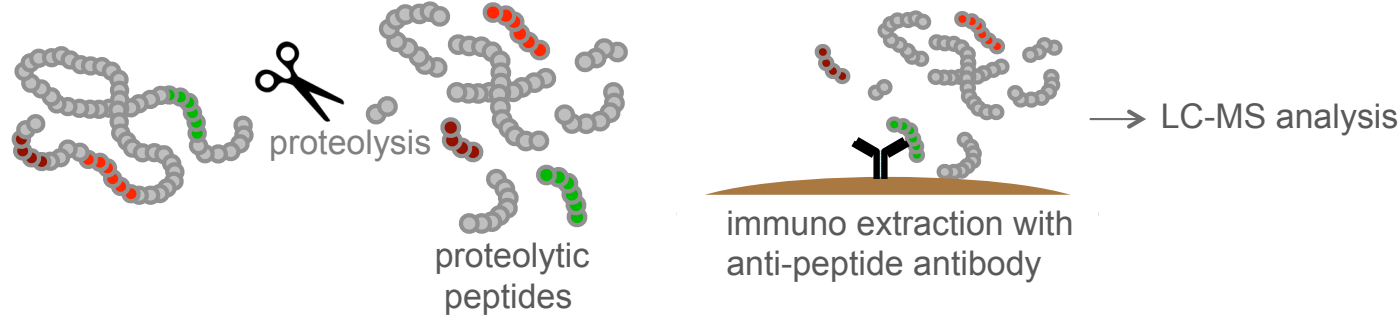

immuno extraction with anti-peptide antibody

\section{Figure 1}



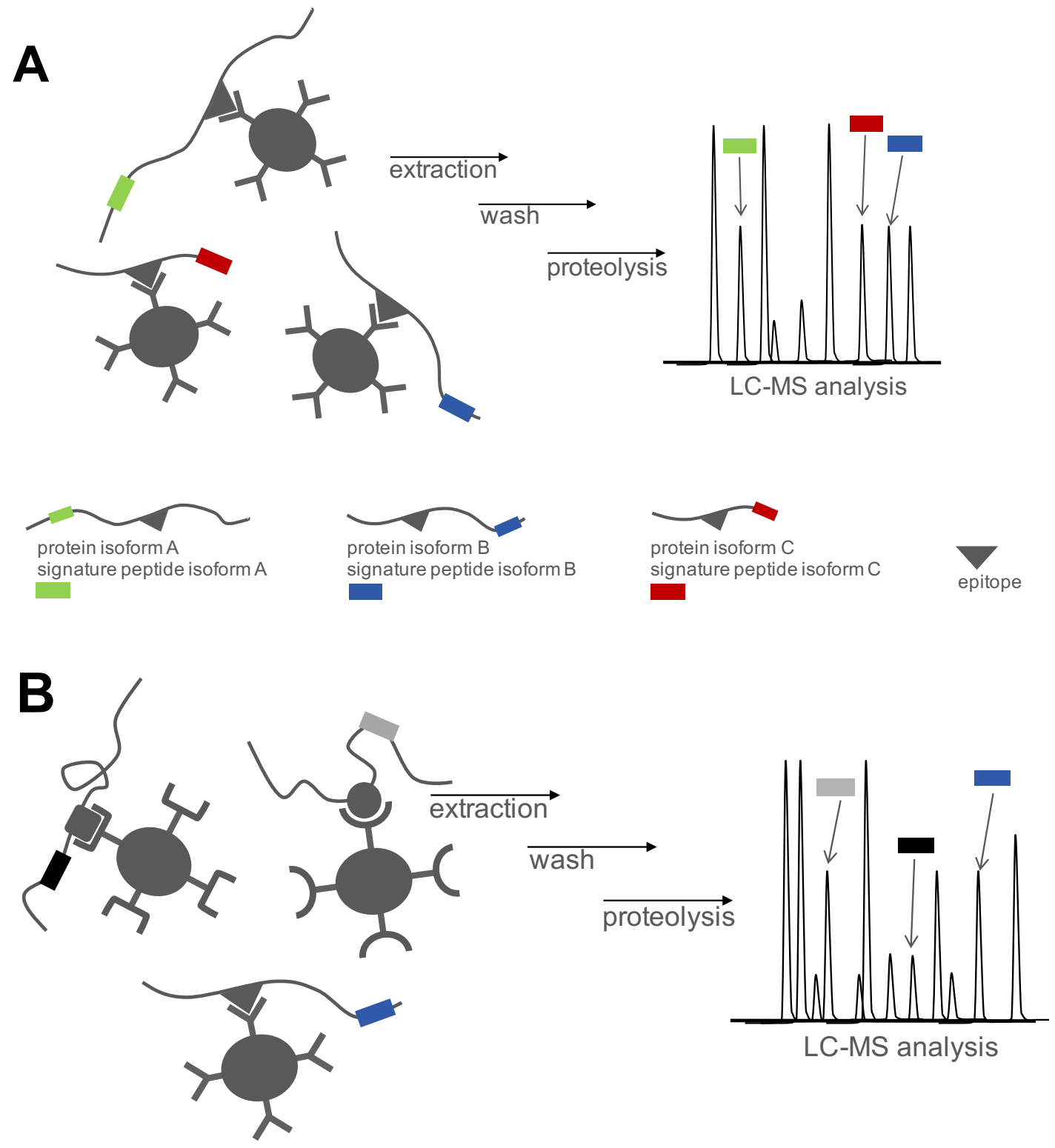

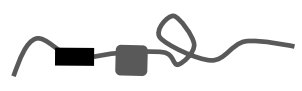

protein 1

signature peptide protein 1

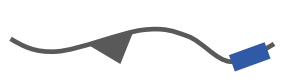

protein 2

signature peptide protein 2

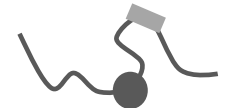

protein 3

signature peptide protein 3

Figure 2 
a)

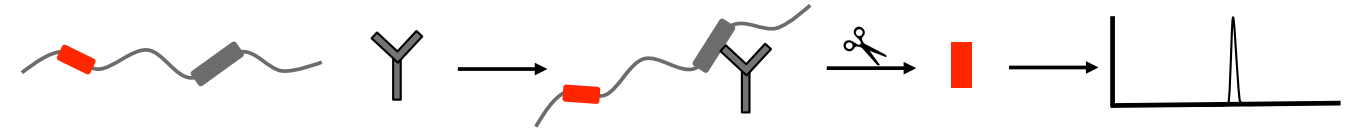

b)

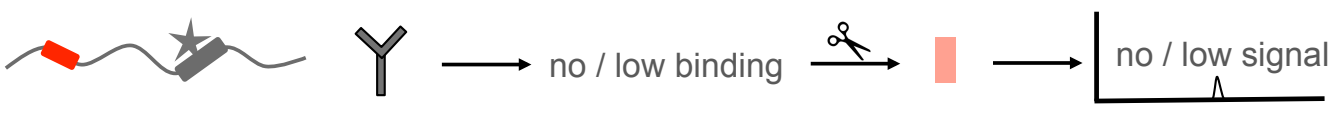

c)

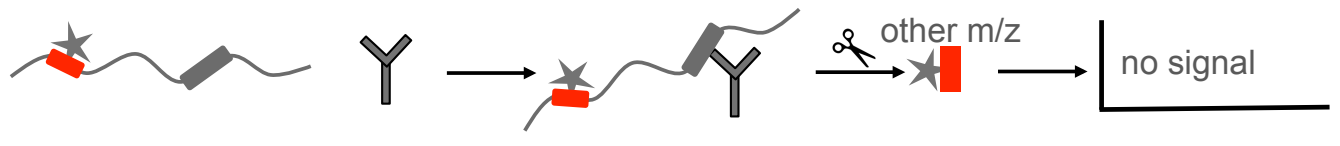

d)

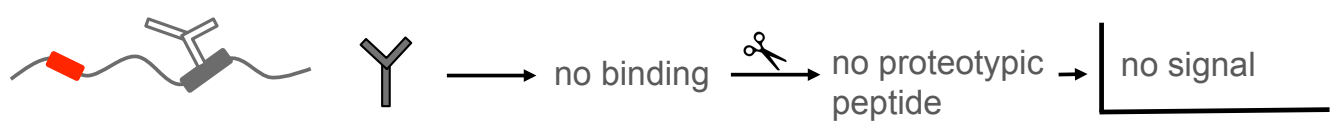

e)

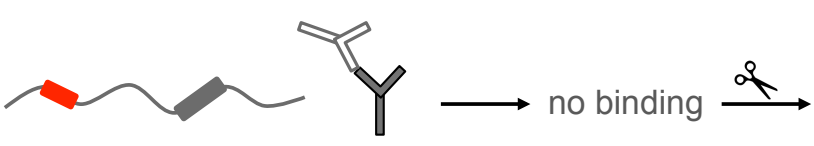

$\rightarrow$ no signal

Figure 3 


\section{References}

[1] Richard van Noorden BM, Regina Nuzzo. The top 100 papers. Nature. 2014;514:550-3.

[2] Anderson NL, Anderson NG. The Human Plasma Proteome. Molecular \& Cellular Proteomics. 2002;1:845-67.

[3] Bantscheff M, Schirle M, Sweetman G, Rick J, Kuster B. Quantitative mass spectrometry in proteomics: A critical review. Anal Bioanal Chem. 2007;389:1017-31.

[4] Anderson L, Hunter CL. Quantitative mass spectrometric multiple reaction monitoring assays for major plasma proteins. Molecular \& Cellular Proteomics. 2006;5:573-88.

[5] Washburn MP, Wolters D, Yates JR. Large-scale analysis of the yeast proteome by multidimensional protein identification technology. Nat Biotech. 2001;19:242-7.

[6] Sturgeon CM, Viljoen A. Analytical error and interference in immunoassay: minimizing risk. Ann Clin Biochem. 2011;48:418-32.

[7] Liu CL, Bowers LD. Immunoaffinity trapping of urinary human chorionic gonadotropin and its high-performance liquid chromatographic-mass spectrometric confirmation. Journal of Chromatography B. 1996;687:213-20. [8] Gam LH, Tham SY, Latiff A. Immunoaffinity extraction and tandem mass spectrometric analysis of human chorionic gonadotropin in doping analysis. J Chromatogr B. 2003;792:187-96.

[9] Kulasingam V, Smith CR, Batruch H, Buckler A, Jeffery DA, Diamandis EP. "Product ion monitoring" assay for prostate-specific antigen in serum using a linear ion-trap. Journal of Proteome Research. 2008;7:640-7.

[10] Lund H, Snilsberg AH, Paus E, Halvorsen TG, Hemmersbach P, Reubsaet L. Sports drug testing using immuno-MS: clinical study comprising administration of human chorionic gonadotropin to males. Anal Bioanal Chem. 2013;405:156976.

[11] van den Broek I, Niessen WMA, van Dongen WD. Bioanalytical LC-MS/MS of protein-based biopharmaceuticals. J Chromatogr B. 2013;929:161-79.

[12] Singh RJ, Hines JM, Lopez MF, Krastins B, Hoofnagle AN. Mass Spectrometric Immunoassay Raises Doubt for the Existence of Parathyroid Hormone Fragment 7-84. Clinical Chemistry. 2015;61:558-60.

[13] Ackermann BL, Berna MJ. Coupling immunoaffinity techniques with MS for quantitative analysis of low-abundance protein biomarkers. Expert Rev Proteomics. 2007;4:175-86.

[14] Lowenthal MS, Gasca-Aragon H, Schiel JE, Dodder NG, Bunk DM. A quantitative LC-MS/MS method for comparative analysis of capture-antibody affinity toward protein antigens. J Chromatogr B. 2011;879:2726-32.

[15] Lund H, Paus E, Berger P, Stenman UH, Torcellini T, Halvorsen TG, et al. Epitope analysis and detection of human chorionic gonadotropin (hCG) variants by monoclonal antibodies and mass spectrometry. Tumor Biology.

2014;35:1013-22. 
[16] Anderson NL, Anderson NG, Haines LR, Hardie DB, Olafson RW, Pearson TW. Mass Spectrometric Quantitation of Peptides and Proteins Using Stable Isotope Standards and Capture by Anti-Peptide Antibodies (SISCAPA). J Proteome Res. 2004;3:235-44.

[17] Becker J, Hoofnagle AN. Replacing immunoassays with tryptic digestionpeptide immunoaffinity enrichment and LC-MS/MS. Bioanalysis. 2012;4:281-90. [18] van Regenmortel MHV. The Recognition of Proteins and Peptides by Antibodies. Journal of Immunoassay. 2000;21:85-108.

[19] Zhao L, Whiteaker JR, Voytovich UJ, Ivey RG, Paulovich AG. AntibodyCoupled Magnetic Beads Can Be Reused in Immuno-MRM Assays To Reduce Cost and Extend Antibody Supply. Journal of Proteome Research. 2015;14:4425-31. [20] Li H, Popp R, Borchers CH. Affinity-mass spectrometric technologies for quantitative proteomics in biological fluids. TrAC Trends in Analytical Chemistry. [21] Dunham WH, Mullin M, Gingras AC. Affinity-purification coupled to mass spectrometry: Basic principles and strategies. Proteomics. 2012;12:1576-90. [22] Yang WC, Kernstock R, Simmons N, Alak A. ELISA microplate: a viable immunocapture platform over magnetic beads for immunoaffinity-LC-MS/MS quantitation of protein therapeutics? Bioanalysis. 2015;7:307-18.

[23] Lund H, Lovsletten K, Paus E, Halvorsen TG, Reubsaett L. Immuno-MS Based Targeted Proteomics: Highly Specific, Sensitive, and Reproducible Human Chorionic Gonadotropin Determination for Clinical Diagnostics and Doping Analysis. Analytical Chemistry. 2012;84:7926-32.

[24] Zhao YM, Chait BT. PROTEIN EPITOPE MAPPING BY MASS-SPECTROMETRY. Analytical Chemistry. 1994;66:3723-6.

[25] Macht M, Marquardt A, Deininger SO, Damoc E, Kohlmann M, Przybylski M. 'Affinity-proteomics': direct protein identification from biological material using mass spectrometric epitope mapping. Anal Bioanal Chem. 2004;378:1102-11.

[26] Kiernan UA. Quantitation of target proteins and post-translational modifications in affinity-based proteomics approaches. Expert Review of Proteomics. 2007;4:421-8.

[27] Torsetnes SB, Broughton MN, Paus E, Halvorsen TG, Reubsaet L.

Determining ProGRP and isoforms in lung and thyroid cancer patient samples:

comparing an MS method with a routine clinical immunoassay. Anal Bioanal Chem. 2014;406:2733-8.

[28] Portelius E, Westman-Brinkmalm A, Zetterberg H, Blennow K. Determination of beta-amyloid peptide signatures in cerebrospinal fluid using immunoprecipitation-mass spectrometry. Journal of Proteome Research. 2006;5:1010-6.

[29] Berna M, Ott L, Engle S, Watson D, Solter P, Ackermann B. Quantification of NTproBNP in rat serum using immunciprecipitation and LC/MS/MS: a biomarker of drug-induced cardiac hypertrophy. Analytical Chemistry. 2008;80:561-6.

[30] Egeland SV, Reubsaet L, Paus E, Halvorsen TG. Dual-immuno-MS technique for improved differentiation power in heterodimeric protein biomarker analysis: determination and differentiation of human chorionic gonadotropin variants in serum. Anal Bioanal Chem. 2016:1-13.

[31] Kumar V, Barnidge DR, Chen L-S, Twentyman JM, Cradic KW, Grebe SK, et al. Quantification of Serum 1-84 Parathyroid Hormone in Patients with

Hyperparathyroidism by Immunocapture In Situ Digestion Liquid 
Chromatography-Tandem Mass Spectrometry. Clinical Chemistry. 2010;56:30613.

[32] Nicol GR, Han M, Kim J, Birse CE, Brand E, Nguyen A, et al. Use of an Immunoaffinity-Mass Spectrometry-based Approach for the Quantification of Protein Biomarkers from Serum Samples of Lung Cancer Patients. Mol Cell Proteomics. 2008;7:1974-82.

[33] Lin D, Alborn WE, Slebos RJC, Liebler DC. Comparison of Protein Immunoprecipitation-Multiple Reaction Monitoring with ELISA for Assay of Biomarker Candidates in Plasma. Journal of Proteome Research. 2013.

[34] Torsetnes SB, Levernæs MS, Broughton MN, Paus E, Halvorsen TG, Reubsaet L. Multiplexing Determination of Small Cell Lung Cancer Biomarkers and Their Isovariants in Serum by Immunocapture LC-MS/MS. Analytical Chemistry. 2014;86:6983-92.

[35] Schoenherr RM, Zhao L, Ivey RG, Voytovich UJ, Kennedy J, Yan P, et al. Commercially available antibodies can be applied in quantitative multiplexed peptide immunoaffinity enrichment targeted mass spectrometry assays. PROTEOMICS. 2016:n/a-n/a.

[36] Kuhn E, Addona T, Keshishian H, Burgess M, Mani DR, Lee RT, et al. Developing Multiplexed Assays for Troponin I and Interleukin-33 in Plasma by Peptide Immunoaffinity Enrichment and Targeted Mass Spectrometry. Clinical Chemistry. 2009;55:1108-17.

[37] Ippoliti PJ, Kuhn E, Mani DR, Fagbami L, Keshishian H, Burgess MW, et al. Automated Microchromatography Enables Multiplexing of Immunoaffinity Enrichment of Peptides to Greater than 150 for Targeted MS-Based Assays. Analytical Chemistry. 2016;88:7548-55.

[38] Olsson N, James P, Borrebaeck CAK, Wingren C. Quantitative proteomics targeting classes of motif-containing peptides using immunoaffinity-based mass spectrometry. Molecular \& Cellular Proteomics. 2012.

[39] Whiteaker JR, Zhao L, Frisch C, Ylera F, Harth S, Knappik A, et al. HighAffinity Recombinant Antibody Fragments (Fabs) Can Be Applied in Peptide Enrichment Immuno-MRM Assays. Journal of Proteome Research. 2014;13:218796.

[40] Percy AJ, Parker CE, Borchers CH. Pre-analytical and analytical variability in absolute quantitative MRM-based plasma proteomic studies. Bioanalysis. 2013;5:2837-56.

[41] Giansanti P, Tsiatsiani L, Low TY, Heck AJR. Six alternative proteases for mass spectrometry-based proteomics beyond trypsin. Nat Protocols.

2016;11:993-1006.

[42] Picard G, Lebert D, Louwagie M, Adrait A, Huillet C, Vandenesch F, et al. $\mathrm{PSAQ}^{\mathrm{TM}}$ standards for accurate MS-based quantification of proteins: from the concept to biomedical applications. JOURNAL OF MASS SPECTROMETRY. 2012;47:1353-63.

[43] Faria M, Halquist MS, Yuan M, Mylott W, Jenkins RG, Karnes HT. Comparison of a stable isotope labeled (SIL) peptide and an extended SIL peptide as internal standards to track digestion variability of an unstable signature peptide during quantification of a cancer biomarker, human osteopontin, from plasma using capillary microflow LC-MS/MS. J Chromatogr B. 2015;1001:156-68. 
[44] Guy MJ, Chen YC, Clinton L, Zhang H, Zhang J, Dong XT, et al. The impact of antibody selection on the detection of cardiac troponin I. Clinica Chimica Acta. 2013;420:82-8.

[45] Streng AS, de Boer D, Bouwman FG, Mariman ECM, Scholten A, van DieijenVisser MP, et al. Development of a targeted selected ion monitoring assay for the elucidation of protease induced structural changes in cardiac troponin T. Journal of Proteomics. 2016;136:123-32.

[46] Streng AS, de Boer D, van Doorn WPTM, Bouwman FG, Mariman ECM, Bekers 0 , et al. Identification and Characterization of Cardiac Troponin $\mathrm{T}$ Fragments in Serum of Patients Suffering from Acute Myocardial Infarction. Clinical Chemistry. 2017;63:563-72.

[47] Ewles M, Mannu R, Fox C, Stanta J, Evans G, Goodwin L, et al. LC-MS/MS strategies for therapeutic antibodies and investigation into the quantitative impact of antidrug-antibodies. Bioanalysis. 2016;8:2565-79.

[48] Hoofnagle AN, Becker JO, Wener MH, Heinecke JW. Quantification of Thyroglobulin, a Low-Abundance Serum Protein, by Immunoaffinity Peptide Enrichment and Tandem Mass Spectrometry. Clin Chem. 2008;54:1796-804. [49] Kushnir MM, Rockwood AL, Roberts WL, Abraham D, Hoofnagle AN, Meikle AW. Measurement of Thyroglobulin by Liquid Chromatography-Tandem Mass Spectrometry in Serum and Plasma in the Presence of Antithyroglobulin Autoantibodies. Clinical Chemistry. 2013;59:982-90.

[50] Woldemariam GA, Butch AW. Immunoextraction-Tandem Mass Spectrometry Method for Measuring Intact Human Chorionic Gonadotropin, Free $\beta$-Subunit, and $\beta$-Subunit Core Fragment in Urine. Clinical Chemistry. 2014;60:1089-97. 